\title{
openaccess
}

\section{Using virtual experiences of older age: exploring pedagogical and psychological experiences of students}

\author{
Joanne Hudson ${ }^{1} \odot$ 0000-0003-4732-8356, Taylor Waters ${ }^{1}$, Marc Holmes ${ }^{1} \oplus$ 0000-0002-8206-5451, Sunil Agris ${ }^{2}$, \\ David Seymour ${ }^{2}$, Laura Thomas ${ }^{3}$, Emily J. Oliver ${ }^{4}$ ๑0 0000-0002-1795-8448 \\ ${ }^{1}$ Swansea University, ${ }^{2}$ Fusion48, ${ }^{3}$ Liverpool John Moores University, ${ }^{4}$ Durham University \\ Corresponding author: joanne.hudson@swansea.ac.uk
}

\begin{abstract}
Fostering intergenerational empathy is vital for creating an age-friendly society and an important aim for Sport and Exercise Science (SES) degree programmes given that graduates are increasingly entering the healthcare workforce supporting older adults (British Association of Sport and Exercise Sciences; BASES, 2018). Interventions to challenge negative stereotypes of ageing, generate empathy for older adults, and help University students gain experience of 'being' an older person have demonstrated mixed success (e.g., Prior \& Sargent-Cox, 2014). Recent studies indicate the promise of virtual reality in this context but do not present conclusive evidence for this effect (e.g., Banakou, Kishore, \& Slater, 2018). Thus this study explored SES students' responses to virtual experiences of being an older person in a workshop. Participants completed the "Become Victor" module of the FrailtySIM ${ }^{\odot}$ application, based on real life experience of an older person in their home, and, a University-developed immersive experience of being an older person in a social situation. Fifty-two students completed questionnaires about their experience of "Become Victor" and 15 students were interviewed (12 in 2 focus groups, 3 individually) about their experiences of both simulations. Data indicated that "Become Victor" offered students insight into being an older person that was "eye-opening" and realistic but frustrating and stressful. The social situation effectively simulated the isolation felt by some older people to an extent, but needed to be more interactive. Students felt that the simulations were important for contextualising previously delivered lecture material on older adults. Future workshop iterations will integrate lecture and virtual experiences using opportunities for student reflection on their experiences.
\end{abstract}

\section{Introduction}

The Western narrative of ageing and older adults is characterised by themes of decay and decline (Gullette, 1997; Hudson, Day, \& Oliver, 2015) and pervasive stereotypes of ageing are predominantly negative (Kotter-Grühn, 2015). This has the potential to impact the behaviour of older adults and how they are treated by others, including ageism and reduced empathy (Kötter-Gruhn, 2015). This is of particular concern given that the global population of older adults is growing, with projected estimates of adults aged 65 and over increasing from 524 million in 2010 to 1.5 billion in 2050 [World Health Organisation (WHO), 2010], relative to a $3 \%$ increase in the overall population (Oliver, Hudson, \& Thomas, 2015).

An important theme in recent research has been understanding how to alter people's perception of older adults and prevent ageism. University students have been the focus of a number of these studies as research indicates that students tend to hold negative attitudes towards
Correspondence

Joanne Hudson (joanne.hudson@swansea.ac.uk)

doi: 10.1255/vrar2018.ch7

Citation: J. Hudson, T. Waters, M. Holmes, S. Agris, D. Seymour, L. Thomas and E.J. Oliver, "Using virtual experiences of older age: exploring pedagogical and psychological experiences of students", in Proceedings of the Virtual and Augmented Reality to Enhance Learning and Teaching in Higher Education Conference 2018, Ed by J. Hudson and R. Kerton. IM Publications Open, Chichester, pp. 61-72 (2019). https://doi. org/10.1255/vrar2018.ch7

\section{(c) 2019 The Authors}

This licence permits you to use, share, copy and redistribute the paper in any medium or any format provided that a full citation to the original paper is given.

Print ISBN: 978-1-906715-30-4 Online ISBN: 978-1-906715-28-1 
older adults (Wurtele \& Maruyama, 2013). This research has primarily targeted students pursuing degrees in Medicine or Professions Allied to Medicine; although they might not interact with older adults on a daily basis and at a personal level, many will do so in their future graduate professions. This is also increasingly the case for graduates of Sport and Exercise Science (SES) degrees, as physical activity is integral for preventing ill health and maintaining both physical and mental health (National Institute for Clinical Excellence (NICE), 2018). As such, it is becoming common place for Sport and Exercise Science graduates to find employment in roles such as Health Promotion Specialist, Exercise Referral Consultant, Clinical Exercise Physiologist, and Clinical Cardiac Physiologist (British Association of Sport and Exercise Sciences; BASES, 2018) where health promotion, maintenance and treatment for older adults are integral aspects. However, to date, there are no studies that have focused on Sport and Exercise Science students.

Numerous studies have designed interventions to modify university students' perceptions of older adults, with varying degrees of success. The methods used in the majority of interventions in these studies are narrative based (e.g., lectures or discussion), or, involve imagined or observed interaction with an older person (e.g., observing an outpatient interaction). Using an imagined interaction with an older person, Prior and Sargent-Cox (2014) demonstrated improvements in males', but not females', age expectations, that were mediated by ageing anxiety. The authors suggested that this interesting gender difference might be attributable to the double standards around ageing in society, leading to greater internalization of negative expectations for ageing in females that are more resistant to change. Evidence presented by Eskildsen and Flacker (2009) showed that a week-long course on ageing improved medical students' attitudes towards, and knowledge about, elderly people. Similarly, following a presentation of positive ageing narratives, images of older adults, and, discursive class activities designed to challenge stereotypes, students' expectations of ageing and stereotypes of ageing showed improvements (Bardach, Gayer, Clinkinbeard, Zanjani, \& Watkins, 2010; Wurtele \& Maruyama, 2013). Interestingly, although Van Winkle et al. (2010) evidenced short-term changes in students' empathy for older people following a workshop intervention, where students observed and reflected on an actor's portrayal of an older adult, these changes were not maintained 7-days post-intervention. We can specu- late that students' passive engagement with the workshop stimulus might not have been sufficient to influence more sustained changes in empathy.

More recently, interventions have begun to extend beyond conventional information delivery to present a greater challenge to students' experiences. For example, Green and Dorr (2016) employed sensory deprivation, where students' vision, hearing, and hand dexterity were impaired whilst performing tasks such as reading, to examine the effects of simulated ageing experiences. Compared with a control group, students in the simulated ageing group reported significantly more favourable attitudes towards older adults but prosocial intentions did not differ between the two groups.

In a similar vein, researchers have also begun to examine the use of virtual reality (VR) to modify agerelated perceptions, for example, by comparing exposure to a VR embodied experience of being an older adult with a traditional perspective-taking experience (reading a newspaper article; Oh, Bailenson, Weisz, \& Zaki, 2016). The embodied, immersive VR experience resulted in greater self-other overlap, suggesting that the experience helped the student to more readily adopt the perspective of an older adult. Although exhibiting similar trends, effects for intention to communicate with older adults and explicit ageism were non-significant. When this embodied virtual experience involved an interactive ball toss game with two virtual older adults, no effects were observed for self-other overlap, intention to communicate with older adults, implicit preference for younger or older adults, or, empathic listening to an older adult. In contrast, Banakou, Kishore, and Slater (2018) evidenced that, compared with a control condition, young adult males (28 of whom were students) who had a VR embodied experience of being Einstein, reported reduced implicit age bias.

Although presenting some preliminary evidence of positive effects, these studies do not offer conclusive evidence supporting the use of VR in this context. In addition, neither of the studies above presented participants with realistic, daily scenarios that older adults might face. Thus the first aim of this study was to examine the effect of realistic virtual ageing experiences on students' perceptions of older adults. Sport and Exercise Science undergraduates were sampled given the lack of previous analysis of their age-related beliefs, and the increasing likelihood of these students working with older adults in their future careers. 
The second aim of this study was to explore the effect of using virtual experiences of being an older adult on student engagement with the learning environment and subject content. Along with preparing students for employment, understanding and enhancing the student experience and student engagement is a priority focus for universities (Shepherd, 2018). This is reflected in the proliferation of recent research interest in this topic (e.g., Reeve \& Lee, 2014; Ouweneel, LeBlanc, \& Schaufeli, 2014; Gunuc \& Kuzu, 2015; Oriol-Granado, MendozaLirab, Covarrubias-Apablazac, \& Molina-López, 2017; Gillet, Morin, \& Reeve, 2017; Ketonen, Dietrich, Moeller, Salmela-Aro, \& Lonka, 2018).

Shepherd discusses the challenges of measuring student engagement that in part stem from its multidimensional nature, making it difficult to clearly define. However, Gunuc and Kuzu (p. 588) employ a definition of student engagement that is useful in this context: the quality and quantity of students' psychological, cognitive, emotional and behavioural reactions to the learning process, as well as to in-class/out-of-class academic and social activities, to achieve successful learning outcomes. This definition highlights not just the cognitive (e.g., learning gained) and behavioural (e.g., practical skills gained) elements of student engagement, but also its psychological and emotional elements that are important for positive learning outcomes.

Preliminary evidence suggests that VR could be particularly effective in this regard. Compared with a traditional narrative-based method of perspective taking, Herrera, Bailenson, Weisz, Ogle, and Zaki (2018) demonstrated that an interactive VR method had a greater impact on emotional, psychological and behavioural responses to homelessness. Participants in the VR condition reported less dehumanisation of homeless people post intervention, their attitudes towards the homeless deteriorated more slowly, and, immediately after the intervention, they reported more personal distress and empathy for the homeless people. More participants in the VR condition also signed an active petition for more affordable housing immediately post-intervention, and, were more anxious, and used more socially-oriented words when writing a letter to an official 2 weeks after the intervention. Effects were not significant across all assessed variables (e.g., self-other connection with the homeless); nevertheless these findings indicate that VR experiences might help to engage participants emotionally and psychologically in the experiences of others who are not like them and whose experiences they do not share in reality. Whether similar engagement is observed in undergraduate Sport and Exercise Science students participating in VR experiences of being an older adult, within the context of a learning experience in Higher Education, remains to be seen.

As alluded to previously, preparing graduates for employment is a key concern for university educators (Wakeham, 2016), including the "soft skills" required in the workplace, such as interpersonal sensitivity (Heaviside, Hudson, \& Manley, 2018). Therefore there is a need for all degree programmes, but especially those that focus on health or sport sciences, to offer opportunities for students to share the experiences of older adults and encourage them to reflect on their perceptions of, and empathy for, older adults, and, their expectations and stereotypes of ageing.

Thus, to summarise, our study aims were twofold: (1) to examine the effect of virtual experiences on students' perceptions of older adults, and, (2) to explore the effect of using virtual experiences of being an older adult on student engagement with the learning environment and subject content. The majority of evidence produced to date has been quantitative which offers insight into what changes occur but does not suggest possible explanations for change or lack of change. Hence, in the present study we used a qualitative approach to gain enhanced understanding of how students engaged with the virtual experiences and how changes in perceptions might have occurred.

\section{Methods Participants}

Participants were 52 final year undergraduate students studying at a UK University (29 males and 10 females; 13 undisclosed), representing $93 \%$ of the cohort registered on an optional multidisciplinary module. The module focused on health related aspects of physical activity and exercise in different clinical and non-clinical population groups, and was delivered through a combination of lectures, seminars, workshops and practical laboratories. The participants were all volunteers who either provided written informed consent after receiving information about the study if they were recruited at the lecture prior to the workshop, or implied consent through completion of the online post-workshop ques- 
tionnaires following receipt of information about the study.

\section{Procedures}

After receiving College Research Ethics Committee approval, participants were recruited in a lecture prior to the delivery of the simulated reality workshop, or at the workshop itself. Students who provided their consent to participate then completed online measures of age stereotype, attitudes towards, and empathy for, older adults (the focus of the Conference presentation was on the qualitative data therefore this paper adopts this focus, but for the interested reader, the additional quantitative measures and results obtained from these are provided in an appendix). In the following week participants took part in a simulated reality workshop as outlined below. After the workshop, they again completed the measures of age stereotype, attitudes towards, and empathy for, older adults, including additional questions that addressed their experiences of the simulated reality workshop. In the two weeks following the workshop, 15 students volunteered and subsequently attended focus groups or interviews that were conducted by one of the graduate teaching assistants who supported the workshop. Based on their availability, 3 students attended an individual semi-structured interview (all male), and 12 attended one of two focus groups comprising 5 males in the first focus group and, 3 males and 4 females in the second.

\section{Simulated reality workshop}

Within seminar groups of 25-30 people, students worked through the workshop activities in small, selfselected groups of 5-6, supported by a module tutor, an external consultant, a teaching technician, and, two graduate teaching assistants. Each workshop, including post-test questionnaire completion and debriefing by the external consultant to conclude, was approximately 2 hours in duration. The workshop included three virtual experiences, each with written instructions and/or a scenario outline to guide students through the activity. An additional physical activity was included that is not discussed here.

Activity 1: The potential older self. Students used the commercially available AgeBooth $^{\odot}$ app to create a visual image of themselves as an older person, using a photograph taken on a handheld tablet device.

Activity 2: Social isolation as an older adult. Students wore a virtual reality headset that allowed them to "take part" in a simulated dinner scene in a restaurant. The scene was filmed using a $360^{\circ}$ camera to offer an immersive experience and the explanatory scenario asked students to imagine themselves as an older relative of the participants in the dinner scene. The participants in the film interacted with each other and acknowledged the student but did not involve them as an interactive member of the scenario.

Activity 3: FrailtySIM "Become Victor" ${ }^{\circ}$. This is a commercially available virtual reality experience based on a real older person's experience of moving around his own home. Via a virtual reality headset, students are guided through an immersive experience of completing a number of everyday tasks (e.g., making a hot drink, answering the door) as would an older person with moderate frailty. For instance, their movement speed and reactions are slowed, their hearing is dulled and vision is blurred.

\section{Measures}

FrailtySIM ${ }^{\circ}$ evaluation. Using a 5-point Likert type scale participants responded to 5 items that assessed ease of use, quality and duration of the experience, relevance to learning outcomes and value in building empathy for older people. They also provided open-ended responses to each of these items and were asked to offer keywords to describe their experience of the simulation. Only the open-ended responses are reported here (see appendix for quantitative responses that are used for app development purposes by Fusion $48^{\circ}$ ).

Focus Group and Interview Schedule. The focus groups and interviews included questions that addressed the following themes: students' experiences of the simulations; benefits and disadvantages for learning; challenges of its use; aspects liked and disliked, and, any effects on personal perceptions of ageing and older people. Participants were also encouraged to add any comments not addressed by these topics, and, the exact order and phrasing of questions was modified in response to group and interview discussions.

\section{Data analysis}

Written responses to open-ended questions about the FrailtySIM ${ }^{\oplus}$ experience were combined with the qualitative data obtained in interviews and focus groups. Interviews and focus groups were audio recorded and transcribed verbatim. Inductive thematic analysis (Braun \& Clarke, 2006) was used to illustrate common themes 
relating to the study's objectives regarding the use of virtual experience, including if and how the intervention had impacted on students' perceptions of older people and ageing. Using this approach, notes and initial impressions were taken by the interviewer during the focus groups. These ideas were then tracked and continually revised throughout transcription and later themes were developed based on underlying meaning. The initial thematic analysis was carried out by the graduate teaching assistant who conducted the interviews and was more familiar with the data. The first author then reviewed this analysis, and, acting as a critical friend, challenged the data interpretation and categorisation and probed explanations for analysis outcomes. On reaching a consensus, the resulting analysis was presented to two of the co-authors who cross-checked the themes identified with data presented to support the themes, thus completing the process of analysis triangulation.

\section{Results}

The inductive thematic analysis produced five key themes that represented the students' perceptions of the virtual experiences: Additional Value, Being Victor, Immersive Experience, Quality, and, Relevance, each comprising a number of different sub-themes, as discussed below. The Being Victor theme referred only to experiences of the FrailtySIM ${ }^{\odot}$ app.

Additional Value This theme was underpinned by three sub-themes: enjoyment, novelty, and, learning. The majority of students discussed the added value that virtual experiences offered above more traditional means of learning (e.g., lectures), which they felt was facilitated in three key ways. The first related to the enhanced opportunities for learning, both reinforcing content previously covered in lectures on the topic: "it helped to understand the psychological barriers a little bit more from a practical point of view..." and, offering opportunities for experiential learning that is less easily gained through other teaching approaches, as a focus group comment illustrates:

...yeah, well because the lecture was about how you can help older adults, and I think experiencing what they experience helps you to understand it more easily, before you've been to the uhh, workshop yeah could easily say 'yeah do this, do this' but without knowing if they are actually able to do it. When you got a better understanding of it I think actually, 'you may not be able to do that, I think you should do this instead'.... Another student talks in literal terms about how the visual and virtual stimuli were, "an eye opener, I think, to experience what, 'cause before, l'd say I had sympathy for older people, but now after that you kind of understand it a bit better, so it puts it into a bigger picture....."

The novelty of the teaching approach contributed to the added value that students discussed, and influenced both student engagement, and enjoyment, as this quotation illustrates, "it was good like, 'cause in previous labs and stuff, we haven't used technology like that it's quite, it was, informative experience and it was also quite fun to use things like that".

Being Victor There were four sub-themes included in this theme: elderly struggles, perspective, emotional response, and student engagement. Students' discussions of Being Victor revealed that with only one or two exceptions, this was a highly emotive and influential experience. For many of these students, this virtual experience made them acutely aware of the elderly struggles that many older adults face on a daily basis that can influence their capacity to be physically active:

...it helps you understand how difficult it might be for someone with the same limitations as Victor to get into physical activity, with his vision being impaired and his mobility being so slow, like it may not be something that he is capable of doing....

For the majority of students this led to enhanced empathy and respect for older adults, as discussed by this student:

I mean, trying to, if you are an old person and you are trying to walk to the shops. And trying to like to stay on your feet more than exercise. I think my grandparents do a bit of golfing. Which is usually about 3 miles for a course, or maybe more or less, like I have big respect for them, they are trying to stay active and I know that they are about that age where all those effects will be happening.

Importantly, the virtual experience of Being Victor offered a new perspective to the students by enabling an experience that was completely unfamiliar to them, "yeah...l liked that, it gave a different perspective, like the kind of rushing and everything, and not being able to keep up". For some students, this not only facilitated a level of engagement with the material, as discussed above, but also facilitated engagement on a personal level, by 
presenting an opportunity to identify with, and even feel like, Victor. Unlike more traditional teaching methods, one student commented that: "it helps you identify with it, and rather than sitting about just learning about it, actually understanding what thousands of people go through...." Focus group discussions between students reinforced this shared experience:

Student 1: it did make you feel like Victor. Like it was realistic...

Student 5: It had the most impact...

For these students, the result was a powerfully emotional experience, "It made me cry", and the majority reported a range of emotions, all of which were negative, for example:

Student 3: the sight was really bad, like you....

Student 4: the speed of the walking, that was really annoying.

Group: yeah, yeah...

Student 5: It was really frustrating.

However, although in the minority, not all students reported having this experience, as one student commented,

yeah, 'cause realistically, if you knew you were going

to be a little slow getting to the door and you hear it. It wasn't a very big flat it was only 5 metres away, you could just say 'yeah hold on a second' or something like that.....

It seems that for this student, the virtual experience did not facilitate the same level of identification with Victor that was reported by other students.

Immersive Experience included two sub-themes: fuller immersion and importance of interaction. The degree of immersion offered by the FrailtySIM ${ }^{\circ}$ app was an important aspect of developing empathy for, and increased awareness of, the challenges faced by some older adults. Similar to comments cited above, one student expressed their view that (bold added):

I thought that was probably the best one of the day,

because...you are that person for a short period of

time. It does make you think. They give you some instructions with it and you have to boil the kettle, then someone knocks on the door on the way to the kettle, then the phone rings, and you miss all this stuff. It just gives you a huge insight into an old person's life. I think that out of all those ones on the day, that was the one that made me respect old people more.

A key element of this immersive experience was the opportunity to interact with the virtual environment, and, some students noted where this was lacking from other activities, detracting from their impact:

...like the menu on the table, if you were able to pick it up and try and look at it closer up. And even if you couldn't see it then, it would still have more of an impact because of the poor vision. If everything was kind of blurred, but if you brought it closer, would it make a difference. So, having a little bit of action with the objects around you, that would make a difference.

It was also clear that an activity that did not offer a personalised experience had little impact on the students: Like that [AgeBooth ${ }^{\odot}$ app], for example, it lacked seriousness, because obviously that wasn't a representation, so that was more just for fun. So if everyone started on that, it may have gotten them to engage a bit more. So it would have been like, this is what we are going to be doing in the next... however long it took. But apart from that, let's be honest, anyone can download an app and be like I am going to look like this at this age...(Laughs)'

Quality was related to the Immersive Experience theme and was discussed in relation to three sub-themes: quality of activities, lack of added value, and, realism. These themes illustrated that, as noted above, whilst the virtual experiences were very real for the majority of students, this was not the case for all of them. Some students did not feel that the scenarios were realistic enough and so did not offer any more learning and experiential value than if they had read about them:

$\mathrm{Hmm}$ it gives a good insight. Yeah it definitely gives insight. But I said it lacks the realism. So, learning wise, yeah, you learn what the elder [sic] people could experience, but not much more so, if I was just about to read a piece of paper or this old person that has cataracts or something like that and just having descriptive text.

One student explained why, in his view, the virtual experiences did not have the same impact for all students: "...yeah, 'cause with VR and anything else, it comes down to interpretation, and everyone interprets things differently". Another commented on the quality of the social isolation scenario and to him, its unconvincing level of realism:

...yeah, but then, but based on the actual scenario it seemed too unrealistic. I know it says imagine you are this guy and you are their grandchildren. But you just think about it logically, 'cause ahh yeah would these people really be my grandchildren. Like 5 people from different ethnicities (laughs). It just didn't seem right. 
Relevance The sub-themes included in this overarching theme were: career, coursework, and, timing, and, were related to the students' academic programme demands and their potential career avenues after graduation. A number of students discussed the value of the workshop in relation to developing career relevant skills, as illustrated by the following example taken from a focus group discussion: "...yeah I think it would definitely help like you planning a programme for an elderly person, that would have definitely been beneficial...."

Nevertheless, given the substantive demands placed on final year undergraduate students, whilst still appreciating its value, some students commented that an experience such as this would be better received earlier on in the degree programme. Students at that point are more open to experiential activities as they are less concerned about time spent away from activities that are not directly related to course assessments:

...in the first or second year yeah...I don't know, maybe if you did it earlier on in the semester maybe...it would be alright but I mean it is a novel way of doing

it, there isn't a better way of experiencing an older person, without waiting another 60 years...

Similarly, whilst students commented on the potential value of the experience in preparing them for careers post-graduation, as the workshop content was not directly assessed, its value in relation to supporting assessed work was perceived as limited:

yeah...if you were going to go into that industry, or be like a doctor or whatever, working with old people, it would be really helpful, like helping you get understanding, but relative to the course and the coursework it didn't have very much of an impact.

\section{Discussion}

The aims of this study were to examine the effect of virtual experiences on students' perceptions of older adults, and, to explore the effect of using virtual experiences of being an older adult on student engagement with the learning environment and subject content. In relation to the first aim, findings demonstrated that virtual experiences can be an effective medium for influencing Sport and Exercise Science undergraduate students' perceptions of older adults. Study findings suggest that the virtual experiences helped students to identify with the needs of, and modify their attitudes towards, older adults.
The students discussed becoming more aware of older adults' experiences and the immersive, and where applicable, interactive environment, offered by virtual reality, helped to facilitate this. The majority of students indicated that the virtual experiences had helped to increase their empathy for older adults, and for some, their experiences led to increased respect for older adults.

These findings add to existing evidence that undergraduate students' attitudes towards, and empathy for, older adults, can be effectively modified through intervention (Banakou et al., 2018; Bardach et al., 2010; Green \& Dorr, 2016; Eskildsen \& Flacker, 2009; Oh et al., 2016; Prior \& Sargent-Cox, 2014; Van Winkle et al., 2010; Wurtele \& Maruyama, 2013). To date, only two of these previous studies have employed virtual reality. Similar to Oh et al.'s (2016) finding of greater self-other overlap through an immersive, embodied experience compared with a traditional approach, our participants reported that the virtual experience helped them to identify with, and indeed briefly become ("you are that person"), an older adult. This was felt by the students to be key to the changes in attitudes they reported. The students' emotional responses to their virtual experiences in this study were particularly striking, as being able to identify with an older adult's experience stimulated strong negative emotions, largely frustration and distress. This supports the emotional impact of virtual reality shown previously when tackling perceptions of the homeless and homelessness (Herrera et al., 2018).

Regarding the second aim of our study, students responses to the virtual experiences suggest that they helped to facilitate increased student engagement. As noted above, students reported meaningful psychological, cognitive and emotional responses to these experiences. This is an important finding as these responses underpin engagement, according to Gunuc and Kuzu (2015). This is supported by recent evidence that virtual reality might be particularly effective in generating empathy and influencing participants' psychological, cognitive, emotional and behavioural responses to a stigmatised group (Herrera et al., 2018). We cannot ignore though, the potential contribution that enjoyment of a novel teaching approach and the technology involved made to student engagement, suggesting that increased exposure might not have the same impact over time. In addition, whilst most students felt the virtual experiences enhanced their learning, this was not a view shared by all, and it appears that not all students' attitudes towards, and empathy for, 
older adults, were positively influenced. It is worth noting here that the participant who reported least benefit of the virtual experiences, commented that it, 'didn't add much more than reading from a textbook', was quite experienced in VR. Thus his experience with better quality VR may have impacted his perception of the realism of the in-house virtual experience. We did not screen for prior experience with VR but this might be a useful addition in subsequent research.

Differences in the degree to which students became immersed in the experiences appear to be one influencing factor here, as do the perceived quality and realism of the scenarios, and, the degree of interaction opportunity they offer participants. It is possible that these individual differences in responding to the virtual reality experiences might go some way to explaining the previously reported largely non-significant effects of virtual reality compared with a traditional perspective taking stimulus (Oh et al., 2016). Future research could explore these individual differences in responding to help all participants to benefit from the experience.

Nevertheless, students' responses during interviews and focus groups suggested that virtual experiences were an effective approach for use in learning and teaching, at least in this context. A number of attributes of the immersive virtual experiences appeared to contribute to this: offering experiential rather than passive learning, often a key method of traditional learning approaches; providing practical reinforcement of ideas encountered in lectures, and, helping to contextualise and highlight the applied relevance of this material.

Lastly, our study enhances a limited body of evidence by focusing on Sport and Exercise Science undergraduates, in comparison with the almost exclusive prior focus on students pursuing medically-related degrees. Unlike these students, Sport and Exercise Science students will not necessarily embark on their degree with the intention of entering into a caring profession or Profession Allied to Medicine. However, their openness to, and engagement with, the current intervention suggests that whilst for many, the ideas raised might not be a priority, the experience nevertheless caused changes in students' awareness of, and beliefs about, older adults.

Potentially these results indicate that virtual experiences might be used to support the development of meta and soft skills such as interpersonal sensitivity and empathy that are valued by employers (Heaviside et al., 2018) and increasingly relevant for graduates from these programmes who are entering into clinical and health related professions (BASES, 2018). In addition, students were able to connect their experiences to future application in careers working with older adults, supporting Heaviside et al.'s suggestion that experiential learning can facilitate the development of students' employability profiles.

In sum, this study makes a novel contribution to a limited evidence base on the use of virtual experiences in learning and teaching, and extends this evidence to focus on Sport and Exercise Science students. We also offer qualitative data to supplement predominantly quantitative evidence, thus providing potential explanations for the links between student experiences and outcomes. Although virtual experiences can be effective in enhancing learning and engagement, and for modifying perceptions of older adults, this is not the case for all students. It might help therefore to increase the personal relevance of the activities for these students and engage in peer-led follow up sessions so that different experiences can be shared, to ensure that more students can gain from the learning and self-development benefits of virtual experiences in $\mathrm{HE}$.

\section{References}

Banakou, D., Kishore, S., \& Slater, M. (2018). Virtually being Einstein results in an improvement in cognitive task performance and a decrease in age bias. Frontiers in Psychology, 9: 917. https://doi. org/10.3389/fpsyg.2018.00917

Bardach, S.H., Gayer, C.C., Clinkinbeard, T., Zanjani, F., $\&$ Watkins, J.F. (2010). The malleability of possible selves and expectations regarding aging. Educational Gerontology, 36(5), 407-424. https://doi. org/10.1080/03601270903212393

BASES (2018). A guide to careers in sport and exercise science. Leeds: BASES.

Braun, V., \& Clarke, V. (2006). Using thematic analysis in psychology. Qualitative Research in Psychology, 3(2), 77-101.

Eskildsen, M.A., \& Flacker, J. (2009). A multimodal aging and dying course for first-year medical students improves knowledge and attitudes. Journal of the American Geriatrics Society, 57(8), 1492-1497.

Gillet, N., Morin, A.J.S., \& Reeve, J. (2017). Stability, change, and implications of students' motivation 
profiles: A latent transition analysis. Contemporary Educational Psychology, 51, 222-239.

Green, S.L., \& Dorr, N. (2016). Changing attitudes toward older adults through a simulated aging exercise: Implications for family therapy. Women \& Therapy, 39(1-2), 69-85. https://doi.org/10.1080/02 703149.2016.1116314

Gullette, M.M. (1997). Declining to decline: Cultural combat and the politics of midlife. Charlottesville: University of Virginia Press.

Gunuc, S., \& Kuzu, A. (2015). Student engagement scale: Development, reliability and validity. Assessment \& Evaluation in Higher Education, 40(4), 587-610. https://doi.org/10.1080/02602938.2014. 938019

Heaviside, H., Hudson, J., \& Manley, A.J. (2018). Bridging the gap between education and employment: A case study of problem-based learning implementation in Postgraduate Sport and Exercise Psychology. Higher Education Pedagogies, 3(1), 463-477.

Herrera, F., Bailenson, J., Weisz, E., Ogle, E., \& Zaki, J. (2018). Building long-term empathy: A large-scale comparison of traditional and virtual reality perspective-taking. Plos ONE, 13(10), e0204494. https://doi. org/10.1371/journal.pone.0204494

Hudson, J., Day, M.C., \& Oliver, E.J. (2015). A 'new life' story or 'delaying the inevitable'? Exploring older people's narratives during exercise uptake. Psychology of Sport \& Exercise, 16, 112-120.

Ketonen, E.E., Dietrich, J., Moeller, J., Salmela-Aro, K., \& Lonka, K. (2018). The role of daily autonomous and controlled educational goals in students' academic emotion states: An experience sampling method approach. Learning and Instruction, 53, 10-20.

Kotter-Grühn, D. (2015). Changing negative views of aging: Implications for intervention and translational research. Annual Review of Geriatrics and Gerontology, 35, 167-186.

NICE (2018). Physical activity: For NHS staff, patients and carers (QS84). UK: NICE.

Oh, S.Y., Bailenson, J., Weisz, E., \& Zaki, J. (2016). Virtually old: Embodied perspective taking and the reduction of ageism under threat. Computers in Human Behavior, 60, 398-410. https://doi. org/10.1016/j.chb.2016.02.007

Oliver, E.J., Hudson, J., \& Thomas, L. (2015). Processes of identity development and behaviour change in later life: Exploring self-talk during physical activity uptake. Ageing and Society, June, 1-19.

Oriol-Granadoa, X., Mendoza-Lirab, M., CovarrubiasApablazac, C-G., \& Molina López, V.M. (2017). Positive emotions, autonomy support and academic performance of university students: The mediating role of academic engagement and self-efficacy. Journal of Psychodidactics, 22(1), 45-53.

Ouweneel, E., LeBlanc, P.M., \& Schaufeli, W.B. (2014). On being grateful and kind: Results of two randomized controlled trials on study-related emotions and academic engagement. The Journal of Psychology, 148(1), 37-60.

Prior, K., \& Sargent-Cox, K.A. (2014). Students' perceptions of ageing: An evaluation of the impact of imagined intergenerational contact and the mediating role of ageing anxiety. Journal of Experimental Social Psychology, 55, 99-104.

Reeve, J., \& Lee, W. (2014). Students' classroom engagement produces longitudinal changes in classroom motivation. Journal of Educational Psychology, 106(2), 527-540.

Shepherd, E. (2018). 27 important questions. Advance HE. July $20^{\text {th }}$.

Van Winkle, L.J., Fjortoft, N., \& Hojat, M. (2012). Impact of a workshop about aging on the empathy scores of pharmacy and medical students. American Journal of Pharmaceutical Education, 76(1), 9.

Wakeham, W. (2016). Wakeham Review of STEM Degree Provision and Graduate Employability. Department for Business, Innovation and Skills. Retrieved from https://www.gov.uk/government/uploads/system/ uploads/attachment_data/file/5185

WHO (2010). World Health Statistics. Geneva: WHO Press.

Wurtele, S.K., \& Maruyama, L. (2013). Changing students' stereotypes of older adults. Teaching in Psychology, 40(1), 59-61. 


\section{Appendix}

\section{Quantitative Measures}

Expectations Regarding Ageing Survey-ERA-12 (Sarkisian, Steers, Hays, \& Mangione, 2005) This scale includes 12 items (4 in each of three subscales: Expectations Regarding Mental Health, Physical Health, and Cognitive Function). The authors developed this scale from the original 38 item measure and demonstrated its reliability (Cronbach's alphas ranging from 0.89 to 0.97 for the three subscales and the whole scale) and temporal stability (intraclass correlation coefficients ranging from 0.78 to 0.94). Participants use a 4-point Likert type scale to respond, anchored by " 1 = Definitely true" and " 4 = Definitely false". Example items include: Every year that people age, their energy levels go down a little more (Physical Health); As people get older they worry more (Mental Health), and, Forgetfulness is a natural occurrence just from growing old (Cognitive Function).

Fraboni Scale of Ageism (FSA; Fraboni, Saltstone, \& Hughes, 1990) This scale includes 29 items structured into three subscales: Antilocution [reflecting antagonism, apathy and misconceptions] with10 items; Discrimination with 9 items, and, Avoidance with 10 items. The authors demonstrated its reliability with Cronbach's alphas ranging from 0.65 to 0.86 for the three subscales and the whole scale, with a 4-point Likert type response scale, anchored by " 1 = Strongly disagree" and " 4 = Strongly agree". Example items include: Many old people just live in the past (Antilocution); Most old people should not be trusted to take care of infants (Discrimination), and, I don't like it when old people try to make conversation with me (Avoidance).

Implicit Association Test of Attitudes Towards Ageing (IAT; Nosek, Greenwald, \& Banaji, 2005) On this computer-based test, participants respond to pairings of face stimuli (i.e., young and old faces) with positively or negatively valenced words (e.g., "Good" and "Bad"). The underlying assumption is that the more closely related a concept (e.g., a young face) with an evaluation (e.g., "Good"), the faster and easier participants are to associate that concept and evaluation. Participants were required to pair both young stimuli with positive items (and old stimuli with negative items) in one block and old stimuli with positive items (and young stimuli with negative items) in another block. The average response latencies in categorising stimuli are compared between two conditions: Young faces and Good items are categorised with one response key and Old faces and Bad items are categorised with another (Condition 1); or Young faces and Bad items are categorised with one response key and Old faces and Good items are categorised with another (Condition 2). Participants who categorise items faster in Condition 1 compared to how fast they categorise items in Condition 2 are considered to have an implicit preference for young people compared to old people (Greenwald, Nosek, \& Banaji, 2003; Westgate et al., 2015). The 7 possible categorisations are: little to no preference; preference for old (slight, moderate or strong), and, preference for young (slight, moderate or strong).

Kiersma-Chen Empathy Scale (K-CES; Kiersma, Chen, Yehle, \& Plake, 2013) This scale includes 15 items that are responded to using a 7-point Likert scale ranging from "1 = Strongly disagree" to "7 = Strongly agree". The authors demonstrated its validity for use with Pharmacy and Nursing students, and, the internal reliability of the scale $(a=0.86)$. An example item is: I am able to value someone else's point of view. We replaced references to Health Care Practitioner, patients and treatment with references to Sport and Exercise Scientists, clients and intervention to ensure relevance for the current participants.

Aging Simulation Experience Survey (ASES; Chen, Kiersma, Yehle, \& Plake, 2015) This scale includes 21 items (8 of which are only administered post experience of the aging simulation), responded to using a 7-point Likert type scale ranging from " 1 = Strongly disagree" to "7 = Strongly agree". In this study, we used 13 of these 21 items, as the remainder were not relevant for Sport and Exercise Science students. The authors developed the scale using qualitative data collected from Pharmacy students over a 4 year period. An example item is: I have respect for older adults, and again, items were re-worded to ensure their relevance to the current student cohort.

Aging Stereotypes and Exercise Scale (AgSES; Chalabaev et al., 2013) This scale includes 12 items constituting three, 4 item factors measuring aging stereotypes related to: Risks of Exercise, Benefits of Exercise, Psychological Barriers to Exercise. Example items from each subscale are, respectively: Older adults do not have sufficient strength to exercise; Exercise raises older adults' spirits (reverse scored), and, Older adults feel capable of exercising (reverse scored) and are responded to using a 7-point Likert type scale ranging from " 1 = Do not 
agree at all" to "7 = Totally agree". The authors confirmed the scale's factor structure, validity, and, temporal and internal consistency (with Cronbach's alphas for each subscale ranging from 0.84 to 0.87 ).

\section{Quantitative Results}

Feedback on the FrailtySIM ${ }^{\odot}$ experience and IAT responses were reviewed using descriptive statistics. Questionnaire data were normally distributed, therefore paired t-tests were used to compare students' responses prior to and following the workshop, using Bonferroni Correction Factor to account for multiple comparisons and an adjusted $p$ value of 0.005 .
Only three attitude variables demonstrated significant (or near significant) changes from pre-post workshop. Stereotyped views of the risks of exercise for older adults increased (AgSES Risks of Exercise; $t(15)=-3.4$, $p=0.004$ ), avoidance of older adults decreased (FSA Avoidance; $t(12)=2.7, p=0.02)$, and, empathy for older adults decreased (KCES; $t(11)=1.83, p=0.095$ ). Table 1 presents the descriptive data for all the age-related variables measured pre and post the simulated reality workshop. Descriptive data for the post experience items on the FrailtySIM ${ }^{\odot}$ questionnaire and the ASES are presented in Table 2.

Table 1. Descriptive statistics (mean \pm standard deviation) for age-related attitude variables measured pre and post simulation workshop.

\begin{tabular}{|l|c|c|}
\hline & Pre-test Mean $( \pm \mathbf{s d})$ & Post-test Mean $( \pm$ sd) \\
\hline ERA-12 & & \\
Mental Health & $10.3(2.4)$ & $11.1(1.3)$ \\
Physical Health & $8.1(2.5)$ & $8.7(2.3)$ \\
Cognitive Function & $10.7(3.1)$ & $10.5(1.4)$ \\
\hline FSA & & \\
Antilocution & $22.9(5.6)$ & $22.8(4.8)$ \\
Discrimination & $23(2.4)$ & $23(2.4)$ \\
Avoidance & $23.3(5.8)$ & $21.5(5.3)$ \\
\hline K-CES & $76.6(6.2)$ & $74.8(6.0)$ \\
\hline ASES & $45.1(6.3)$ & $45.5(5.6)$ \\
\hline AgSES & & \\
Benefits & $23.1(3.0)$ & $23.6(2.7)$ \\
Risks & $12.5(5.3)$ & $20.3(4.7)$ \\
Psychological Barriers & $16.5(3.6)$ & $16.1(4.4)$ \\
\hline
\end{tabular}

Note: ERA-12 = Expectations Regarding Ageing Survey - ERA-12 (Sarkisian et al., 2005); FSA = Fraboni Scale of Ageism (Fraboni et al., 1990); K-CES = Kiersma-Chen Empathy Scale (Kiersma et al., 2013); ASES = Aging Simulation Experience Survey (Chen et al., 2015); AgSES = Aging Stereotypes and Exercise Scale (Chalabaev et al., 2013).

Table 2. Descriptive statistics (mean \pm standard deviation) for post simulated reality workshop evaluation measures.

\begin{tabular}{|l|c|l|c|}
\hline \multicolumn{1}{|c|}{ ASES Item $(\mathrm{n}=\mathbf{5 1})$} & Mean $(\mathbf{\pm} \mathrm{sd})$ & \multicolumn{1}{|c|}{ FrailtySIM ${ }^{\odot}$ Items $(\mathrm{n}=\mathbf{5 2})$} & Mean $( \pm \mathrm{sd})$ \\
\hline I experienced frustration when I lost an ability & $5.6(1.5)$ & Ease of set up and use & $4.7(0.4)$ \\
\hline $\begin{array}{l}\text { My attitude toward older adults did not } \\
\text { change as a result of this experience }\end{array}$ & $3.7(1.8)$ & $\begin{array}{l}\text { Quality of virtual reality } \\
\text { experience }\end{array}$ & $4.3(0.5)$ \\
\hline $\begin{array}{l}\text { I plan to be patient with older adults when I } \\
\text { am working }\end{array}$ & $5.8(1.1)$ & $\begin{array}{l}\text { Duration of virtual reality } \\
\text { experience }\end{array}$ & $4.1(0.9)$ \\
\hline $\begin{array}{l}\text { I experienced frustration when I was unable } \\
\text { to complete a task easily }\end{array}$ & $5.4(1.4)$ & Relevance to learning outcomes & $4.5(0.7)$ \\
\hline $\begin{array}{l}\text { I plan to provide assistance to older adults in } \\
\text { my future career }\end{array}$ & $5.2(1.3)$ & Helpfulness in building empathy & $4.6(0.6)$ \\
\hline
\end{tabular}




\section{References}

Chalabaev, A., Emile, M., Corrion, K., Stephan, Y., Clément-Guillotin, C., Pradier, C., \& d'ArripeLongueville, F. (2013). Development and validation of the Aging Stereotypes and Exercise Scale. Journal of Aging and Physical Activity, 21(3), 319-334.

Chen, A.M.H., Kiersma, M.E., Yehle, K.S., \& Plake, K.S. (2015). Impact of an aging simulation game on pharmacy students' empathy for older adults. American Journal of Pharmaceutical Education, 79(5), Article 65.

Fraboni, M., Saltstone, R., \& Hughes, S. (1990). The Fraboni Scale of Ageism (FSA): An attempt at a more precise measure of ageism. Canadian Journal on Ageing, 9(1), 56-66.
Kiersma, M.E., Chen, A., Yehle, K.S., \& Plake, K.S. (2013). Validation of an empathy scale in pharmacy and nursing students. American Journal of Pharmaceutical Education, 77(5), Article 94.

Nosek, B.A., Greenwald, A.G., \& Banaji, M.R. (2005). Understanding and using the Implicit Association Test: II. Method variables and construct validity. Personality and Social Psychology Bulletin, 31(2), 166-180.

Sarkisian, C.A., Steers, W.N., Hays, R.D., \& Mangione, C.M. (2005). Development of the 12-item Expectations Regarding Aging survey. The Gerontologist, 45(2), 240-248. 